\title{
Handwritten Character Recognition using Neural Network with comparing Result with Feature Extraction Techniques
}

\author{
Shraddha Kunkunkar ${ }^{1}$, Preeti Motwani ${ }^{2}$, Vaibhav Palav ${ }^{3}$, Vijay Kawade ${ }^{4}$ \\ Department of Electronics \& Telecommunications, Lokmanya Tilak College of Engineering and Technology, \\ Navi Mumbai, India ${ }^{1,2,3,4}$
}

\begin{abstract}
This paper research is based on handwritten character recognition. For character recognition to achieve, better accuracy is important. By using the neural network and feature extraction technique, the recognition is achieved. This paper proposes the HCR using Alphabets \& Digit characters by using feature extraction techniques.
\end{abstract}

Keywords: Back Propagation Neural Network, Classification Rate, Conventional, Directional, Gradient (sobel operator) Feature Extraction Technique, MPLN Using Back Propagation, Recognition Rate.

\section{INTRODUCTION}

Character recognition is definitely the application area where it is becoming more and more important in modern world. To recognize the handwritten character is the difficult task for the computers so the researchers have been involved in handwritten character recognition.

Handwritten character recognition is not a new technology also there are some tedious methods used earlier for recognizing the characters but the goal of the system is to achieve good accuracy because human beings are not able to recognize every handwritten character without any vocabulary text.

There are several techniques for recognizing the characters. Among this, neural network has made their first significance contribution in engineering [7] [8]. By using the back propagation neural network recognition, accuracy is achieved [1]. In this paper describing the recognition of alphabet and digit character is done using neural network with twelve directional feature extraction technique. In this paper, initially gradients feature extracting from the normalization image using sobel and Gaussian mask technique is done. And after that feature is extracted with the twelve directional feature extraction using gradients. The results are experimented on matlab and the result is based on twelve directional techniques which is having the better accuracy.

\section{LITERATURE REVIEW}

This paper describes review of the techniques used in the previous papers. By online and offline characters recognition is the method for the optically sensed document text to translate in human readable characters to machine readable code with this it achieve 95\% accuracy [11]. Lot of work has done in feature extraction technique each pixel is extracted and achieved high accuracy [3]. In this paper it describes higher resolution on both magnitude and angle of the directional strokes in character image [4].The effect of stroke direction caused by shape normalized-cooperated gradient feature extraction provide higher recognition [12]. In [13] developed directional feature extraction technique it gives the character which normalized it takes the each character in specific direction and achieve accuracy rate. In achieving better accuracy modified quadratic extraction technique has developed with 95.42\% accuracy [14]. These papers proposed only for feature extraction for character recognition for using the real application the system should be in adaptable and recognition rate should be more for this case the system is use called neural network [7][8][9]. In this network the feed forward back propagation neural network is achieve fastest recognition rate with the training time 98.12\% [1][6][15]. Neural network describes adaptive character recognition feature extraction [10][15].

From this above literature review it describes the feature extraction and neural back propagation technique which is used for recognition. This system proposes for describing the twelve directional technique in detail using the characters. In this section describes the database for character, section III describes system model, section IV feature extraction technique, and section $\mathrm{V}$ describes Back propagation neural technique with multi perceptron neural network VI conclusion. 
UGC Approved Journal

IJIREEICE

\section{International Journal of Innovative Research in} Electrical, Electronics, Instrumentation and Control Engineering

ISO 3297:2007 Certified

Vol. 5, Issue 7, July 2017

\section{SYSTEM MODEL}

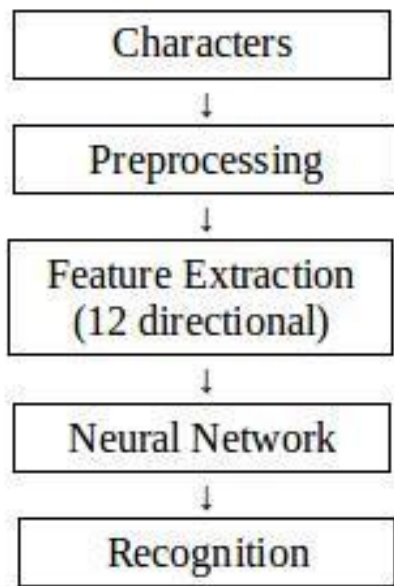

Figure 1: Character Reorganization Model

\subsection{Database}

In this paper, database used are for Alphabets and digits. Each character is having 10 samples (A-Z), (0-9). This database is having the variations in shape, different size, thickness. This database is used for neural network for training and testing the data.

\subsection{Preprocessing}

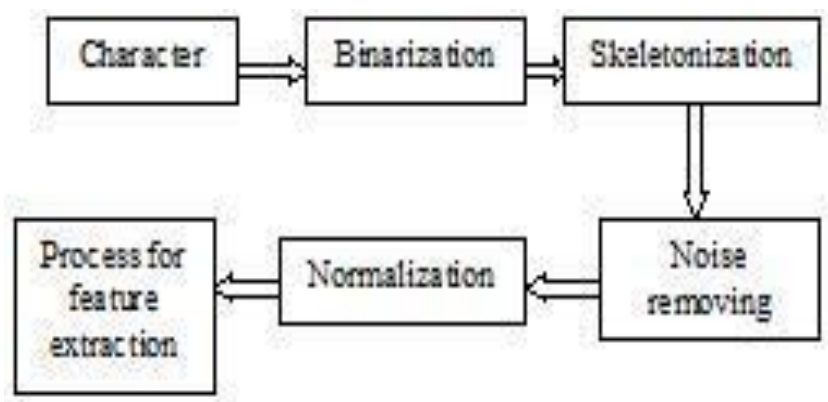

Figure 2: Preprocessing Model

The preprocessing performing important role in pattern recognition for matching the character and the pattern should be in distortion less Character. In this method the raw data and brings into the specific format for recognize the character fastly.

\subsubsection{Binarization}

In the pattern matching if the image or pattern in the grey scale then this image or character is converted into the binary image (black and white) by using the global image threshold. So that recognize the character in to black and white colour is become easy and it increases the accuracy.

\subsubsection{Skeletonization}

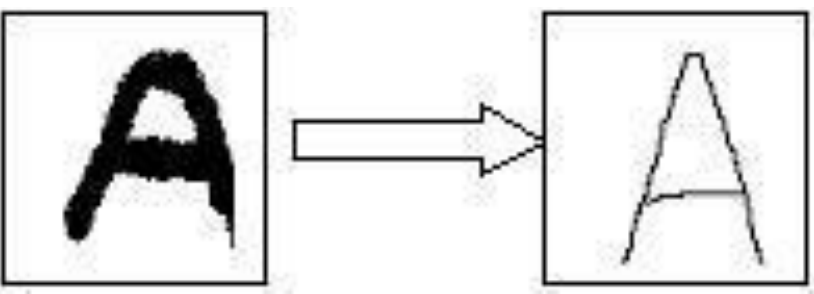

Figure 3: Thinning 
UGC Approved Journal

IJIREEICE

Vol. 5, Issue 7, July 2017

The Skeletonization is the process of removing unwanted pixel. It perform thinning, remove the trunk of character by using spurring and brings into standardize size. Skeletonization is obtain from geometrical and topological property.

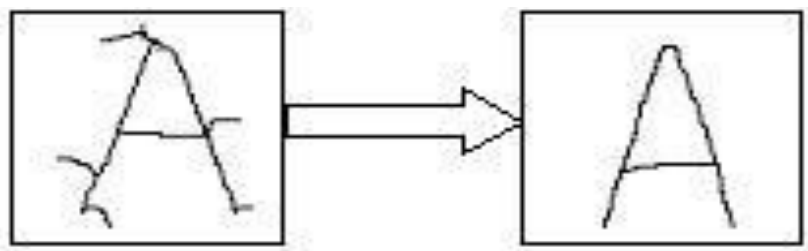

Figure 4: Spurring

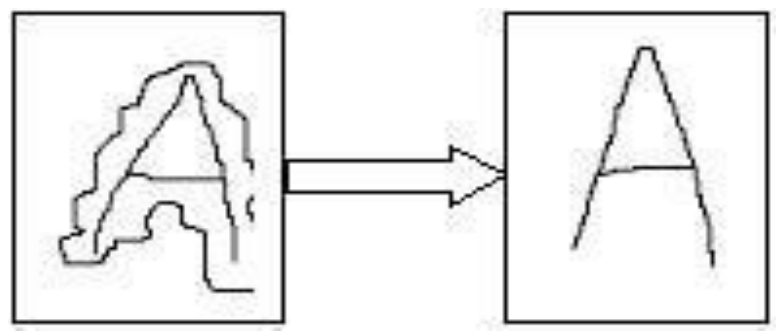

Figure 5: Topology Method

\subsubsection{Noise Removing}

In this technique it removes the noise which present in character. This noise is obtained from the electronic instrument like optical scanning device, writing instrument. Noise can remove by image processing method like filtering method.

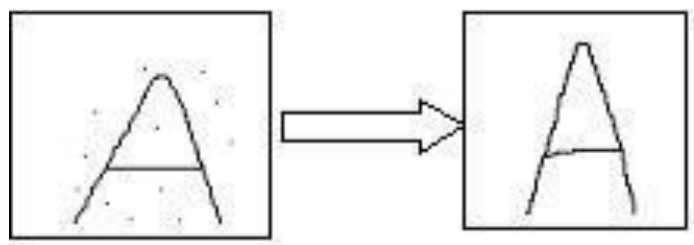

Figure 6: Noise Removing Method

\subsubsection{Normalization}

The normalization is the part of preprocessing where It converts the random character into the standard or specific size. It consist fragmentation. Fragmentation it reduce s the un-necessary part of the character where it has two types horizontal and vertical fragmentation.

\subsubsection{Horizontal Fragmentation- It Removes the Upper and Bottom Un- Necessary Part}
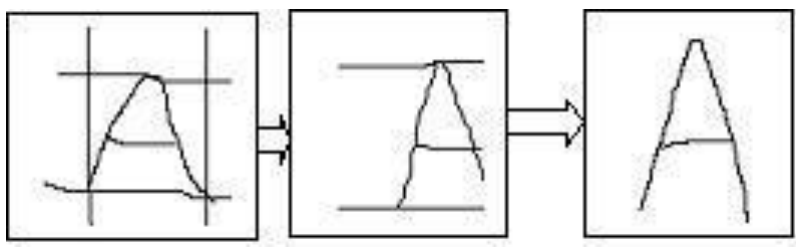

Figure 7: Horizontal Fragmentation

\subsubsection{Vertical Fragmentation- It Removes the Left and Right Un-Necessary Part}

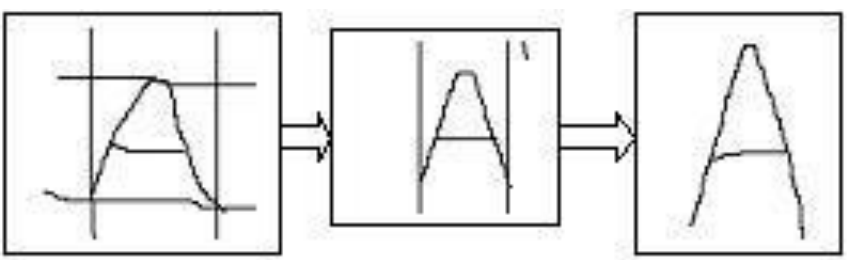

Figure 8: Vertical Fragmentation 
UGC Approved Journal

IJIREEICE

ISO 3297:2007 Certified

Vol. 5, Issue 7, July 2017

\section{FEATURE EXTRACTION}

Feature extraction is used for pattern recognition and data analysis. Feature extraction has many different techniques to extract the feature for recognition. This reduce computational requirement which is based on two types of features.

\subsection{Stastical}

It distribution of pixel in terms of stastical.

\subsection{Structural}

This features based on topological and geometrical property. This paper propose the three feature extraction method for character recognition

\subsection{Conventional Feature Extraction}

In conventional method for each pixel it has horizontal and vertical border. In that case if a line passes through a pixel then corresponding pixel will be given value (1) or (0) but it takes lot of time to extracting the character.

\subsection{Gradient Feature Extraction}

Gradient feature is used for character recognition [11] the gradient feature is more robust against contour noise and applies to gray scale image as well as binary image. It has sobel operator which applies the result on image point which depends on the colour of intensity. The gradient of two variable functions where the each image point describes by intensity of the image if it has constant image then the image intensity is zero if it has on edge vector then it has higher values .

To extend the gradient feature, $3 * 3$ sobel operator are used to the gradient components in horizontal and vertical direction respectively
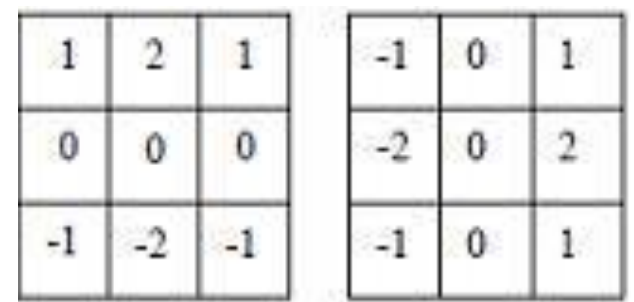

Figure 9: Horizontal Mask and Vertical

$$
\begin{gathered}
\text { Mask Gx }=g y(i, j)=f(i-1, j+1)+2 f(i, j+1)+f(i+1, j+1) \\
-f(i-1, j-1)-2 f(i, j-1)-f(i+1, j-1) \\
\text { Gy=gy }(i, j)=f(i-1, j-1)+2 f(i-1, j)+f(i-1, j+1) \\
-f(i+1, j-1)-2 f(i+1, j)-f(i+1, j+1) \\
\operatorname{grad}=G y / G x=\tan -1[g h(i . j) / g y(i, j)
\end{gathered}
$$

\subsection{Directional Feature Extraction}

This technique is used recognize the characters. after normalizing the character image into standard size. Each pixel applies directional planes with each recording the local stroke components in a specific direction. This technique applies on the gradient by using the sobel mask operator to recognize the character directionally. This paper describes the four. Eight and twelve directional Directional comparing in detail in terms of classification time, Training time

\subsubsection{For Four Directional}

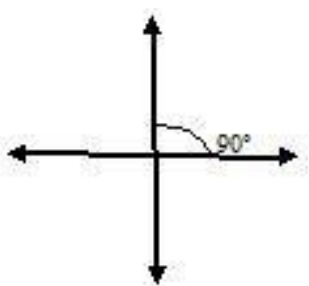

Figure 10: Graphical Representation of Four Directional 
UGC Approved Journal

IJIREEICE

International Journal of Innovative Research in Electrical, Electronics, Instrumentation and Control Engineering

ISO 3297:2007 Certified

Vol. 5, Issue 7, July 2017

Table 1: Gradient Values for Four Directional

\begin{tabular}{|l|l|}
\hline Gradient Values & Direction \\
\hline $\mathrm{g}=-1$ & 0 \\
\hline $0 \leq \mathrm{g} \leq \Pi / 2$ & 1 \\
\hline$\Pi / 2 \leq \mathrm{g} \leq \Pi$ & 2 \\
\hline$\Pi \leq \mathrm{g} \leq 3 \Pi / 2$ & 3 \\
\hline $3 \Pi / 2 \leq \mathrm{g} \leq 2 \Pi$ & 4 \\
\hline
\end{tabular}

4.5.2 For Eight Directional

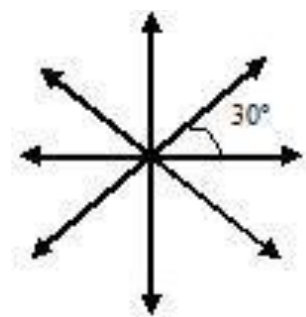

Figure 11: Graphical Representation of Eight Directional

Table 2: Gradient Values for Four Directional

\begin{tabular}{|l|l|}
\hline Gradient Values & Directions \\
\hline $\mathrm{g}=-1$ & 0 \\
\hline $0 \leq \mathrm{g} \leq \Pi / 4$ & 1 \\
\hline$\Pi / 4 \leq \mathrm{g} \leq \Pi / 2$ & 2 \\
\hline$\Pi / 2 \leq \mathrm{g} \leq 3 \Pi / 4$ & 3 \\
\hline $3 \Pi / 4 \leq \mathrm{g} \leq \Pi$ & 4 \\
\hline$\Pi \leq \mathrm{g} \leq 5 \Pi / 4$ & 5 \\
\hline $5 \Pi / 4 \leq \mathrm{g} \leq 3 \Pi / 2$ & 6 \\
\hline $3 \Pi / 2 \leq \mathrm{g} \leq 7 \Pi / 2$ & 7 \\
\hline $7 \Pi / 2 \leq \mathrm{g} \leq 2 \Pi$ & 8 \\
\hline
\end{tabular}

4.5.3 For Twelve Directional

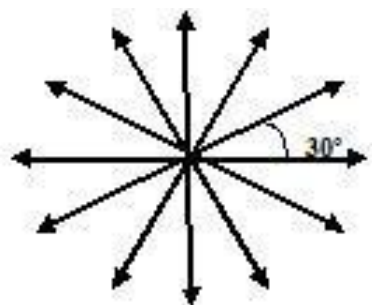

Figure 12: Graphical Representation of Twelve Directions

Table 3: Gradient Values for Four Directional

\begin{tabular}{|l|l|}
\hline Gradient Values & Directions \\
\hline $\mathrm{g}=-1$ & 0 \\
\hline $0 \leq \mathrm{g} \leq \Pi / 6$ & 1 \\
\hline$\Pi / 6 \leq \mathrm{g} \leq \Pi / 3$ & 2 \\
\hline$\Pi / 3 \leq \mathrm{g} \leq \Pi / 2$ & 3 \\
\hline$\Pi / 2 \leq \mathrm{g} \leq \Pi$ & 4 \\
\hline$\Pi \leq \mathrm{g} \leq 2 \Pi / 3$ & 5 \\
\hline $2 \Pi / 3 \leq \mathrm{g} \leq 5 \Pi / 6$ & 6 \\
\hline $5 \Pi / 6 \leq \mathrm{g} \leq \Pi$ & 7 \\
\hline$\Pi \leq \mathrm{g} \leq 7 \Pi / 6$ & 8 \\
\hline $7 \Pi / 6 \leq \mathrm{g} \leq 4 \Pi / 3$ & 9 \\
\hline $3 \Pi / 3 \leq \mathrm{g} \leq 5 \Pi / 2$ & 10 \\
\hline $5 \Pi / 3 \leq \mathrm{g} \leq 11 \Pi / 6$ & 11 \\
\hline $11 \Pi / 6 \leq \mathrm{g} \leq 2 \Pi$ & 12 \\
\hline
\end{tabular}


This result shows the directional pattern values. using the gradient extraction technique for each pixel.

\section{BACK PROPAGATION NEURAL NETWORK}

Back propagation neural network is used for recognition system [10] [13] In Back propagation the recognition task take place with in less time for that purpose system we to be trained by using database.

Back propagation is a systematic method for training multi-layer artificial neural networks. The multilayer has no. of. inputs A1, A2 .....An are called association units and their task is to extract specific, localized featured from the input images this model name as multilayer perceptron neural network MPLN with some additional, fixed, pre-processing. Perceptrons mimic the basic idea behind the visual system. They were mainly used in pattern recognition even though their capabilities extended a lot more. [8][9].

Backward propagation of errors is a common method of training neural networks. Back propagation is a systematic method for training multi-layer artificial neural networks. It is a multi-layer forward network using extend gradientdescent based delta learning rule. Back propagation provides a computationally efficient method for changing the weights in feed forward network. The aim of this network is to train the net to achieve a balance between the input and output with provide good responses to the similar [10]

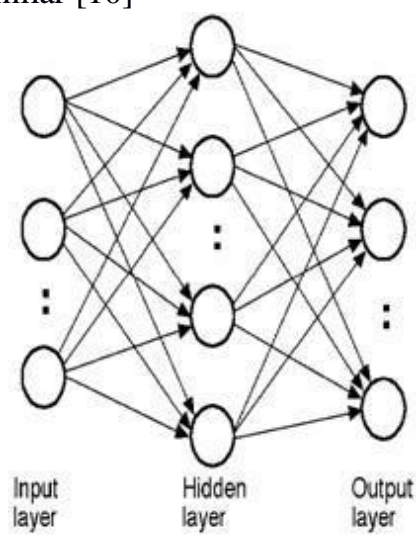

Figure 13

Learning in Back Propagation

There are two types of rule,

\subsection{Sequential Rule or Pre Pattern Rule}

This rule is used for free parameter and pattern classification for real applications.

\subsection{Batch Learning and Pre-Epoch Method}

This rule used for time period basis which has entire training set it is used for nonlinear regression.

\section{EXPERIMENTING AND ANALYSIS}

This system proposes experiment on $32 * 32$ Kannada and English characters and digits character and comparing the result on four, eight, and twelve types of directions. In this paper the parameter will be observing are

Table 4: Experimenting and Analysis Parameters

\begin{tabular}{|c|}
\hline Parameters \\
\hline 32*32Input characters \\
\hline (Trad.,Gradient,4,8,12 directional) \\
\hline Input to single layerd or MPLN \\
\hline No. of hidden layers \\
\hline Training time \\
\hline Testing time \\
\hline Recognition rate \\
\hline Performance on accuracy \\
\hline
\end{tabular}


UGC Approved Journal

IJIREEICE

\section{International Journal of Innovative Research in} Electrical, Electronics, Instrumentation and Control Engineering

ISO 3297:2007 Certified

Vol. 5, Issue 7, July 2017

A MATLAB 7.8.0(R2009a)

Eile Edit Debug Parallel Desktop Window Help

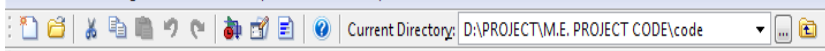

Shortcuts 제 How to Add 지 What's New

\begin{tabular}{|c|c|c|c|}
\hline Current Directory & $+\square \pi x$ & Command Window & Workspace $\quad+1 \square \star x$ \\
\hline 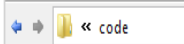 & 、费 & Test Data Creation Done. & 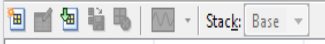 \\
\hline [] Name & Date Modified $v$ & $\begin{array}{l}\text { Class }=1: 25 \\
\text { Class }=2: 25\end{array}$ & Name 4 \\
\hline$\boxplus$ trainKanG.mat & $7 / 10 / 144: 38 \mathrm{PM}$ & Class $=3: 25$ & \\
\hline 画 trainKanD,mat & 7/10/14 4:38 PM & Class $=4: 25$ & \\
\hline$\boxplus$ trainKanT.mat & 7/10/14 4:37 PM & Class $=5: 25$ & \\
\hline$\boxplus$ networkEngD8-20.mat & 7/10/14 4:36 PM & Class $=6: 25$ & \\
\hline$\boxplus$ networkEng.mat & 7/10/14 4:36 PM & Class $=7: 25$ & \\
\hline$\boxplus$ networkDigito8-20.mat & 7/10/14 4:34 PM & Class $=8: 25$ & \\
\hline$\boxplus$ networkDigitD4-20.mat & 7/10/14 4:34 PM & Class $=9: 25$ & \\
\hline$\boxplus$ networkDigitD-20.mat & 7/10/14 4:33 PM & Class $=10: 25$ & \\
\hline $\mathbb{\boxplus}$ networkDigitG-20.mat & 7/10/14 4:31 PM & Per $=100$ & \\
\hline$\boxplus$ networkDigitT-20.mat & 7/10/14 4:31 PM & Test Data Creation Done. & \\
\hline 田 trainEngD8.mat & 7/10/14 4:29 PM & Class $=1: 48$ & \\
\hline$\boxplus$ trainDigitT.mat & 7/10/14 4:28 PM & Class $=2: 44$ & III \\
\hline $\mathbb{\boxplus}_{\text {teestKanT.mat }}$ & $\begin{array}{l}\text { 7/10/142:22 PM } \\
7 / 10 / 142: 21 \mathrm{PM}\end{array}$ & $\begin{array}{l}\text { Class }=3: 40 \\
\text { Class }=4: 39\end{array}$ & 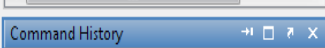 \\
\hline $\begin{array}{l}\boxplus \text { testKanD4.mat } \\
\boxplus \text { testKanD8.mat }\end{array}$ & $\begin{array}{l}\text { 7/10/142:21 PM } \\
7 / 10 / 142: 20 \mathrm{PM}\end{array}$ & $\begin{array}{l}\text { Class }=4: 39 \\
\text { Class }=5: 42\end{array}$ & trainDataKan $\left(10,{ }^{\prime}\right.$ D $\left.8^{\prime}\right)$ \\
\hline 岜 testKanD.mat & $7 / 10 / 142: 20 \mathrm{PM}$ & Class $=6: 41$ & neuralKan $\left(' T^{\prime}, 20\right)$ \\
\hline 田 testKanG.mat & 7/10/14 2:20 PM & Class $=7: 44$ & neuralKan ('G', 20) \\
\hline TestKan.m & 7/10/14 2:19 PM & Class $=8: 49$ & neuralKan('D', 20) \\
\hline theateTestDataKan.m & 7/10/14 2:17 PM & Class $=9: 44$ & neuralKan ('D4', 20) \\
\hline testKan.Tmat & 7/10/142:15 PM & Class $=10: 44$ & neuralKan('D8', 20) \\
\hline & 7/10/142:13 PM & $\begin{array}{l}\text { Per }=87 \\
f x \gg\end{array}$ & neuralkan('DS', 20 ) \\
\hline $\begin{array}{l}\boxplus \text { testEngD.mat } \\
\mathbb{H}_{\text {testEngD8.mat }}\end{array}$ & $\begin{array}{l}\text { 7/10/142:10 PM } \\
7 / 11 / 142: 10 \text { PM }\end{array}$ & $f \underset{x}{x} \gg$ & recogDigitall ('G' 20$)$ \\
\hline 岗 testengD 48 .mat & $7 / 10 / 142: 09$ PM & & recogDigitall ('D4',20) \\
\hline testengD4.mat & $7 / 10 / 142: 09$ PM & & recogDigitAll ('D8',20) \\
\hline testEngG.mat & 7/10/14 2:08 PM & & recogDigitAll $(' D$ ' 20$)$ \\
\hline$\boxplus$ testEngT.mat & 7/10/142:08 PM & & recogDigitAll (' $D$ ', 25$)$ \\
\hline TestEng.m & 7/10/14 2:07 PM & & recogDigitAll ('D', 20 ) \\
\hline & ^ & & recongEngAll (' T', 20) \\
\hline
\end{tabular}

1 Start

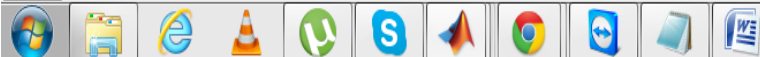

Result of traditional for digits

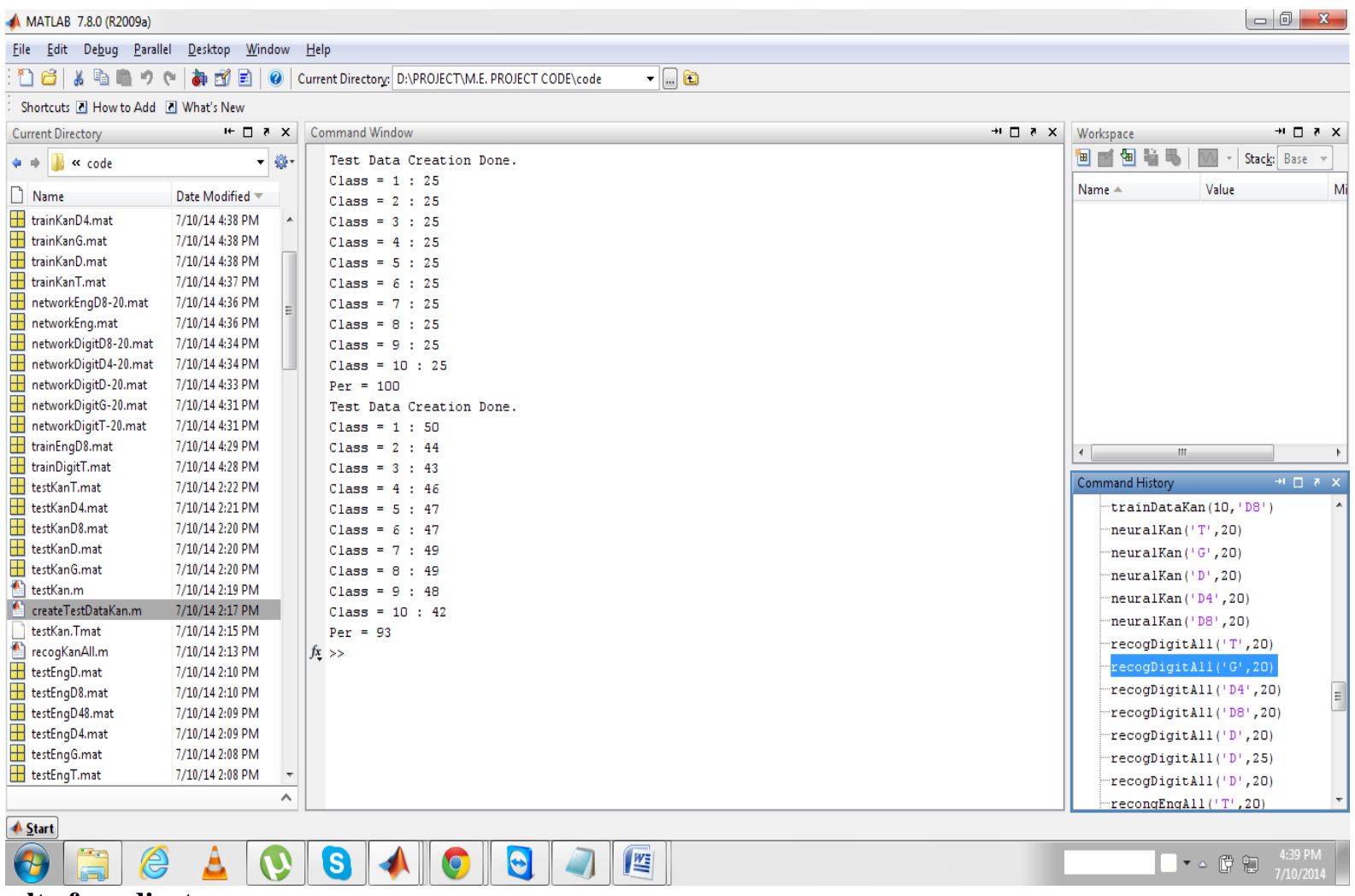

Result of gradient 
UGC Approved Journal

IJIREEICE

International Journal of Innovative Research in Electrical, Electronics, Instrumentation and Control Engineering

ISO 3297:2007 Certified

Vol. 5, Issue 7, July 2017

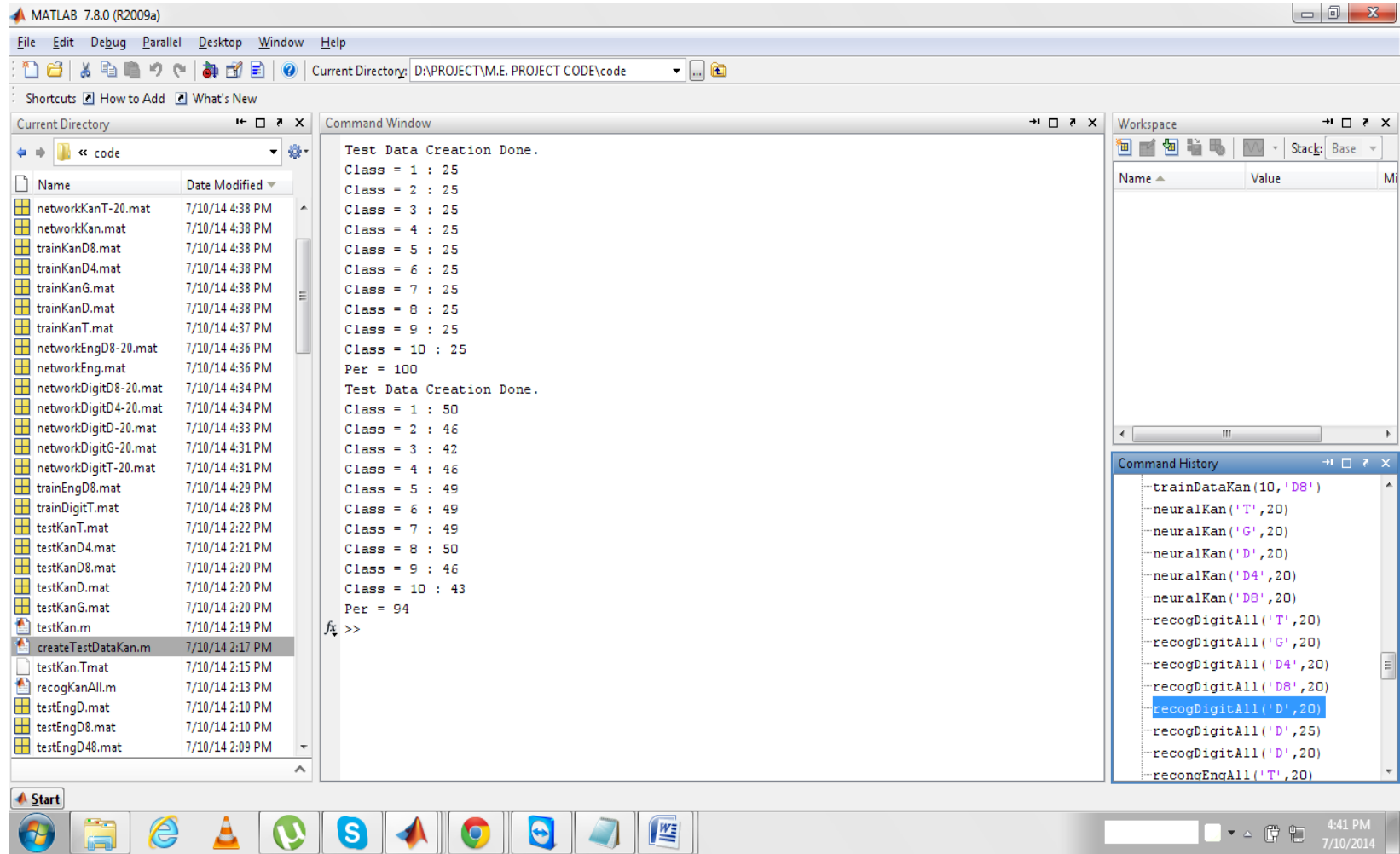

Result of Directional

\begin{tabular}{|l|l|l|l|}
\hline Rate & Traditional & gradient & Directional \\
\hline Recognition rate & $87 \%$ & $93 \%$ & $94 \%$ \\
\hline
\end{tabular}

A MATLAB 7.8.0 (R2009a)

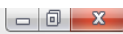

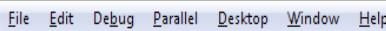

\begin{tabular}{|c|c|c|c|c|c|c|}
\hline \multirow{2}{*}{\multicolumn{7}{|c|}{ 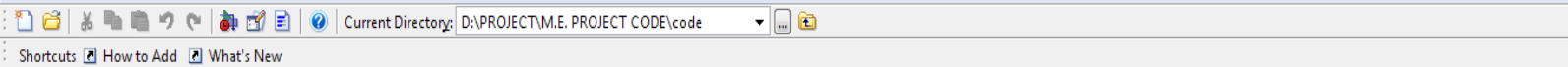 }} \\
\hline & & & & & & \\
\hline Current Directory & \multicolumn{2}{|c|}{$+\square \times x$} & \multicolumn{2}{|l|}{ Command Window } & $+1 \square \times x$ & Workspace $\quad \rightarrow 1 \square x$ \\
\hline \multirow{2}{*}{$\Leftrightarrow \Rightarrow$ u code } & & 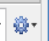 & \multirow{2}{*}{\multicolumn{2}{|c|}{$\begin{array}{l}\text { Test Data Creation Done. } \\
\text { Class }=1: 25\end{array}$}} & A & 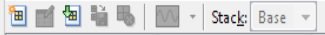 \\
\hline & Date Modified & & & & & \begin{tabular}{|l|l} 
Name $\triangle$ & Value \\
\end{tabular} \\
\hline \multirow{2}{*}{$\begin{array}{l}\text { 田 testDigitG.mat } \\
\text { testDigitT.mat }\end{array}$} & 7/10/14 4:39 PM &. & \multicolumn{2}{|l|}{ Class $=3: 25$} & & \\
\hline & 7/10/14 4:38 PM & & \multicolumn{2}{|l|}{ Class $=4: 25$} & & \\
\hline 画 networkKanT-20.mat & 7/10/14 4:38 PM & 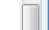 & \multicolumn{2}{|l|}{ Class $=5: 25$} & & \\
\hline 田 networkKan.mat & 7/10/14 4:38 PM & & \multicolumn{2}{|l|}{ Class $=6: 25$} & & \\
\hline \multirow{2}{*}{$\begin{array}{l}\text { 崮 trainKanD8,mat } \\
\text { trainKanD4,mat }\end{array}$} & 7/10/14 4:38 PM & $=$ & \multicolumn{2}{|l|}{ Class $=7: 25$} & & \\
\hline & 7/10/14 4:38 PM & & \multicolumn{2}{|l|}{ Class $=8: 25$} & & \\
\hline 崮 trainKanG.mat & 7/10/14 4:38 PM & & \multicolumn{2}{|l|}{ Class $=9: 25$} & $=1+1+2+2$ & \\
\hline trainKanD,mat & 7/10/14 4:38 PM & 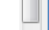 & \multirow{2}{*}{\multicolumn{2}{|c|}{ Class $=10: 25$}} & & \\
\hline 思 trainKanT.mat & 7/10/14 4:37 PM & & & & & \\
\hline 崮 networkEngD8-20.mat & 7/10/14 4:36 PM & & Class $=12: 25$ & & & \\
\hline \multirow{2}{*}{$\begin{array}{l}\boxplus \text { networkEng.mat } \\
\text { 思 }{ }_{\text {networkDigitD8-20.mat }}\end{array}$} & 7/10/14 4:36 PM & & Class $=13: 25$ & & & \\
\hline & 7/10/14 4:34 PM & & Class $=14: 25$ & & & $1 \longdiv { 1 1 }$ \\
\hline 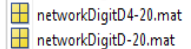 & $\begin{array}{l}\text { 7/10/14 4:34 PM } \\
\text { 7/10/14 4:33 PM }\end{array}$ & & $\begin{array}{l}\text { Class }=15: 25 \\
\text { Class }=16: 25\end{array}$ & & & Command History \\
\hline $\begin{array}{l}\text { 崮 netwokrkUlgitiv-20.mat } \\
\text { networkDigitG-20.mat }\end{array}$ & 7/10/14 4:31 PM & & $\begin{array}{l}\text { Class }=16: 25 \\
\text { Class }=17: 25\end{array}$ & & 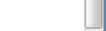 & recogDigitall(' $D$ ', 20$)$ \\
\hline $\mathbb{\boxplus}$ networkDigitT-20.mat & 7/10/14 4:31 PM & & Class $=18: 25$ & & & recongEngAl1 ('T', 20) \\
\hline 面 trainEngD8.mat & 7/10/14 4:29 PM & & Class $=19: 25$ & & & recogEngAl1 ('T', 20) \\
\hline $\mathbb{\boxplus}_{\text {trainDigitT.mat }}$ & 7/10/14 4:28 PM & & Class $=20: 25$ & & & recogEngAl1 ('G', 20$)$ \\
\hline 界 testKanT.mat & 7/10/142:22 PM & & Class $=21: 25$ & & & recogEngA11 ('D4', 20) \\
\hline 界 testKanD4.mat & $\begin{array}{l}\text { 7/10/142:21 PM } \\
7 / 10 / 142: 20 \mathrm{PM}\end{array}$ & & Class $=22: 25$ & & & recogEngA11 ('D 48 ', 20) \\
\hline 岗 testKanD. mat & $\begin{array}{l}\text { 7/10/1142:20 PM } \\
\text { 7/10/142:20 PM }\end{array}$ & & $\begin{array}{l}\text { Class }=23: 25 \\
\text { Class }=24: 25\end{array}$ & & & recogEngA11 ('D8', 20) \\
\hline 画 testKanG.mat & $7 / 10 / 142: 20$ PM & & Class $=25: 25$ & & & recogEngA11 ('D', 20) \\
\hline TestKan.m & 7/10/14 2:19 PM & & Class $=26: 25$ & & & recogRanA11 ('T', 20) \\
\hline createTestDataKan.m & 7/10/142:17 PM & & Per $=100$ & & & trainDataKan $\left(10, \mathrm{~T}^{\prime}\right)$ \\
\hline testKan.Tmat & 7/10/14 2:15 PM & & Test Data Creation Done. & & & neuralkan $(' T ', 20)$ \\
\hline 1. recogKanAll.m & $7 / 10 / 142: 13$ PM & & Class $=1: 45$ & & & recogKanA11 (' T', 20) \\
\hline 画 testengD.mat & 7/10/14 2:10 PM & - & Class $=2: 47$ & & & recogKanA11 ('G', 20$)$ \\
\hline & & $\hat{\imath}$ & Class $=3: 48$ & & - & recogKanA11('D', 20) \\
\hline A S tart & & & & & & sen \\
\hline (2) & 昰 & Q & 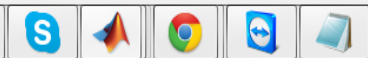 & WE & & 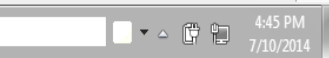 \\
\hline
\end{tabular}


UGC Approved Journal

IJIREEICE

International Journal of Innovative Research in Electrical, Electronics, Instrumentation and Control Engineering

ISO 3297:2007 Certified

Vol. 5, Issue 7, July 2017

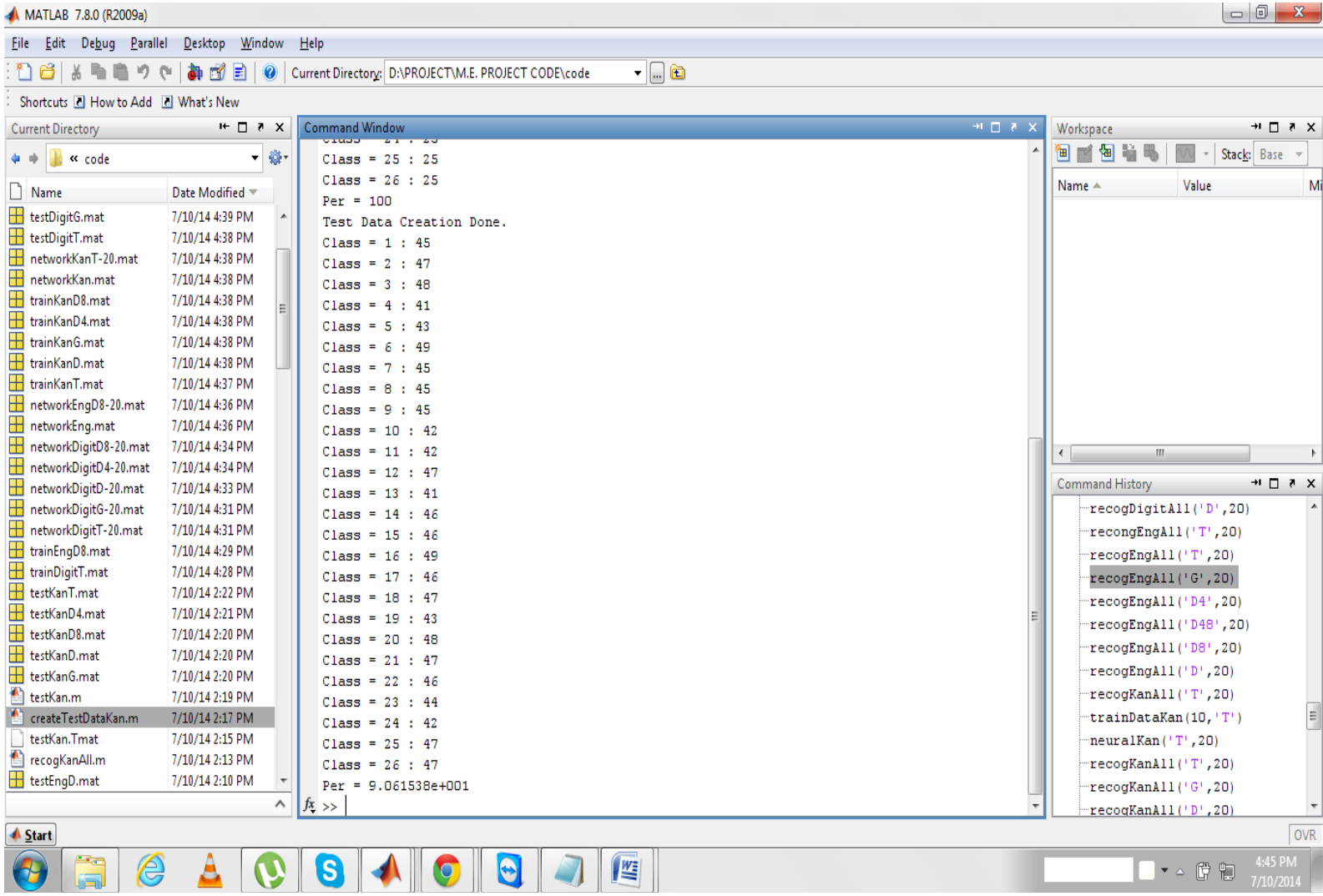

\section{Result of gradient}

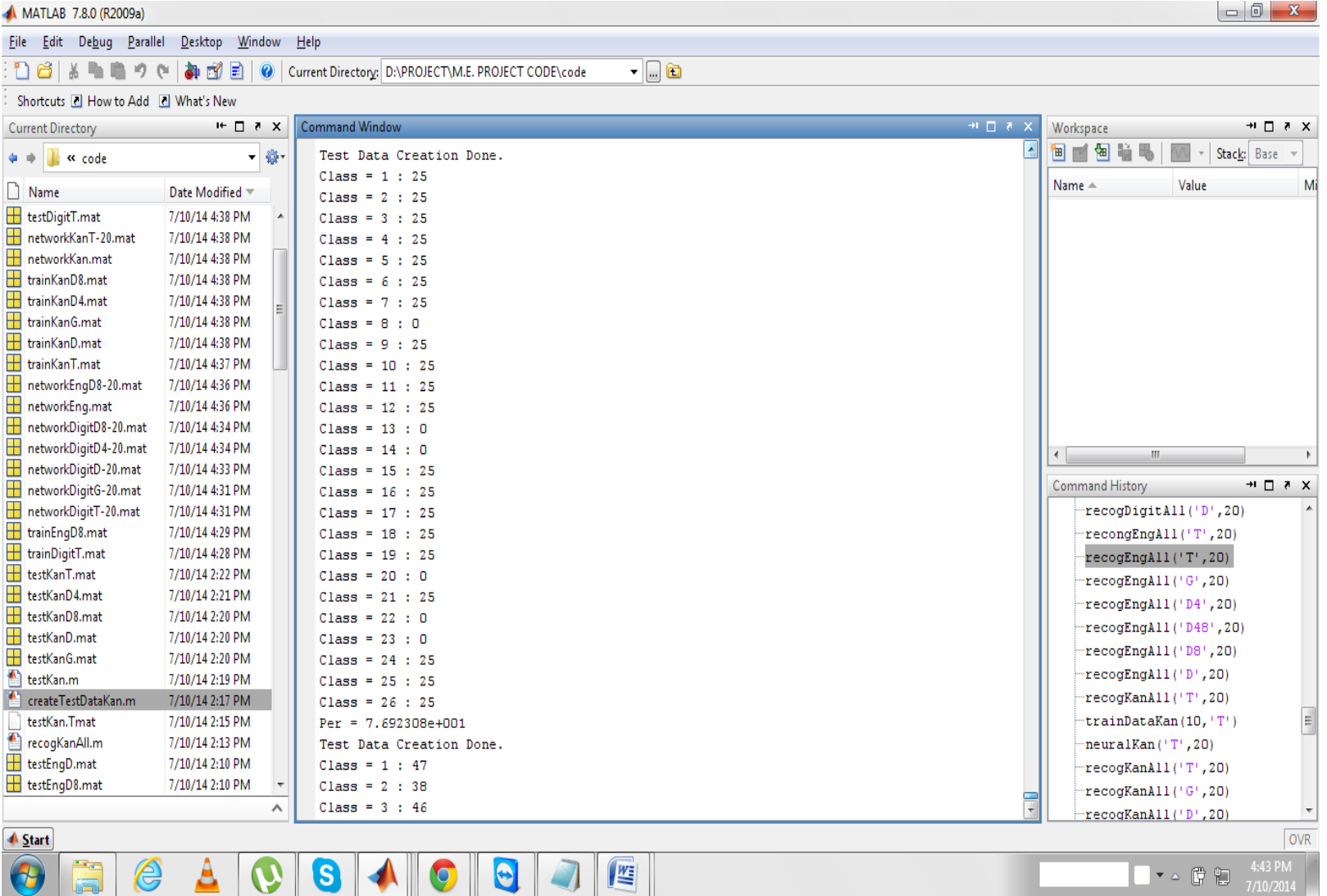


UGC Approved Journal

IJIREEICE

International Journal of Innovative Research in Electrical, Electronics, Instrumentation and Control Engineering

ISO 3297:2007 Certified

Vol. 5, Issue 7, July 2017

4. MATLAB 7.8.0 (R2009a)

File E⿱diti Debug Parallel Desktop Window Help

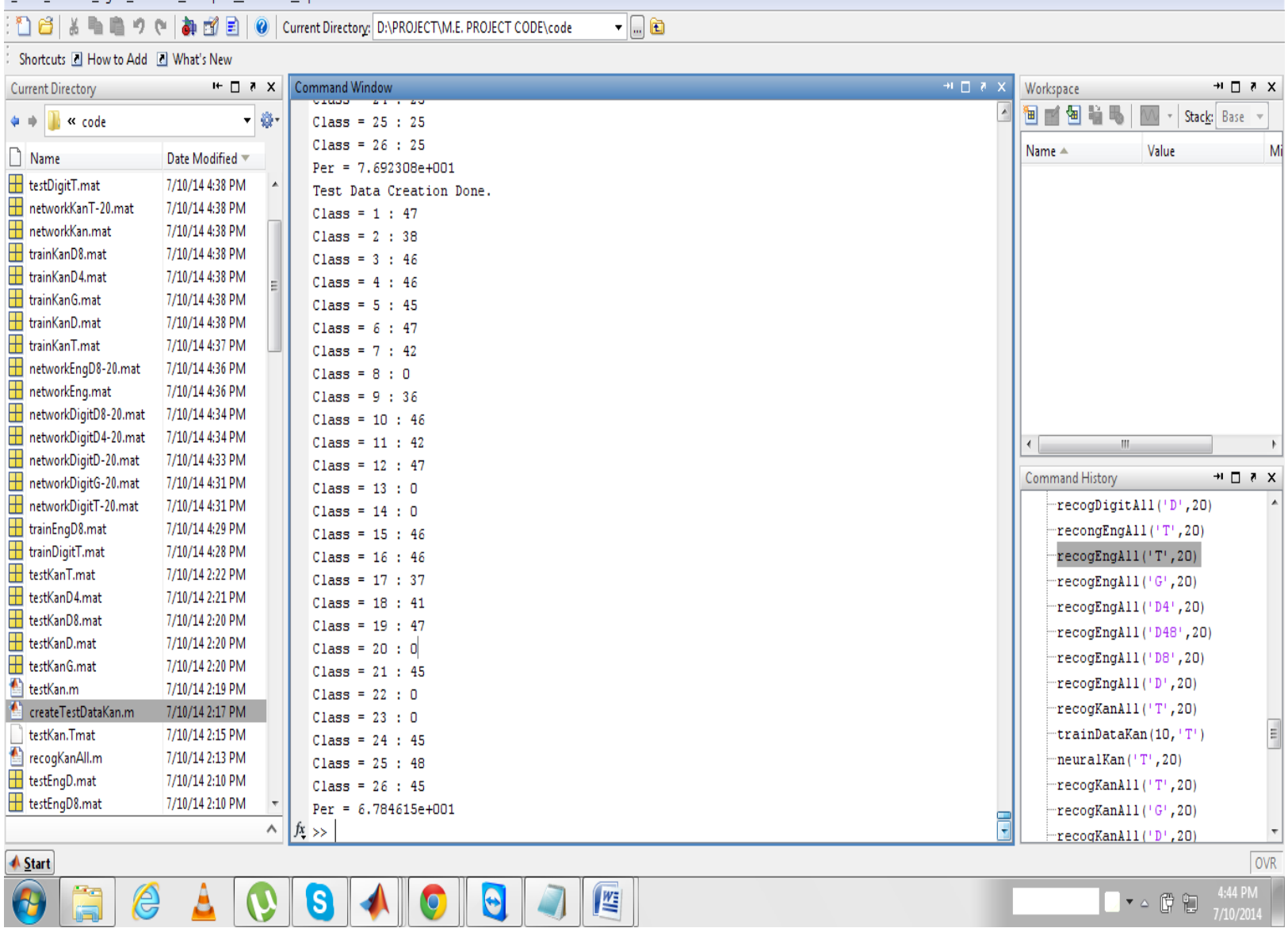

\section{Result of traditional}

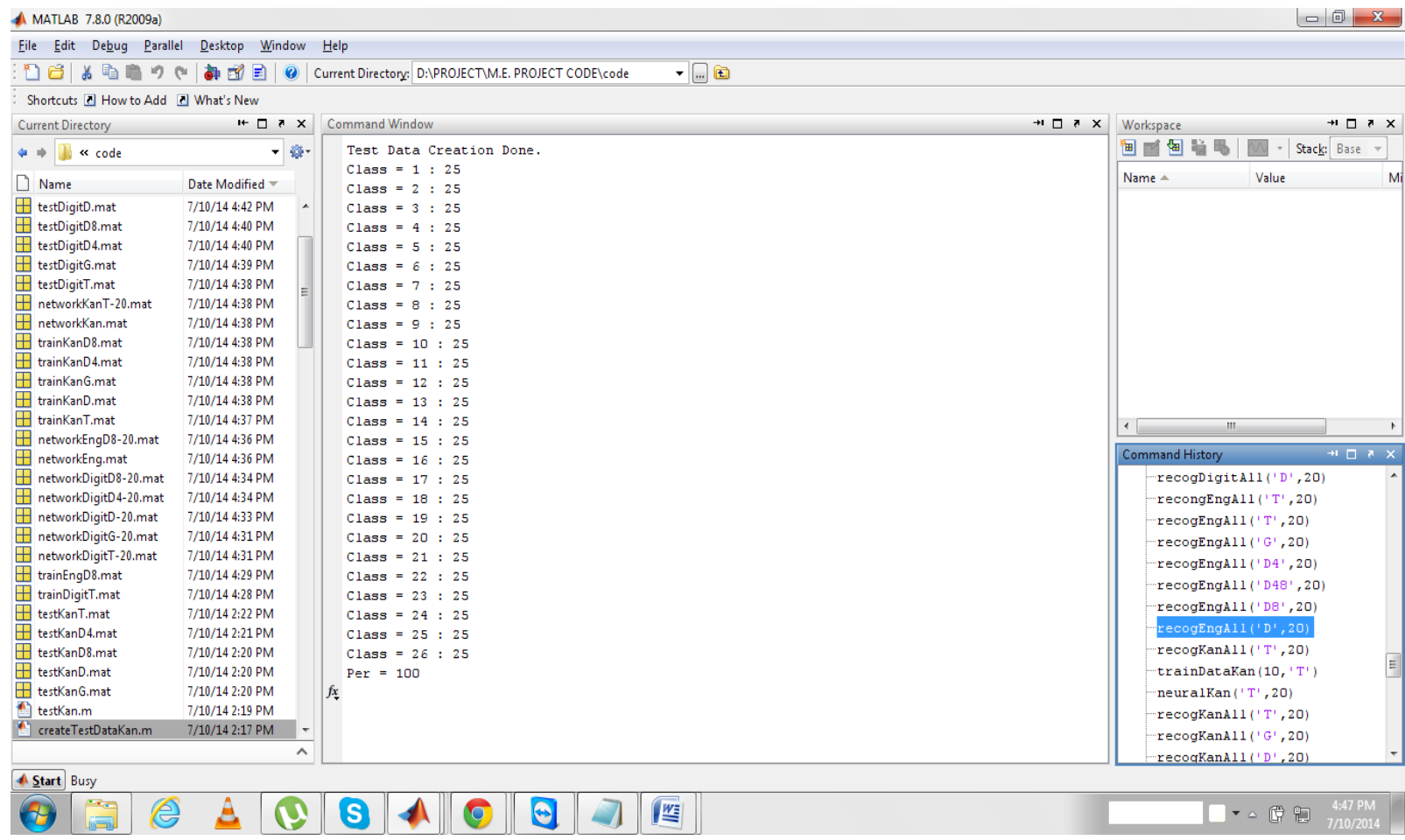

\section{Result of directional}


UGC Approved Journal

IJIREEICE

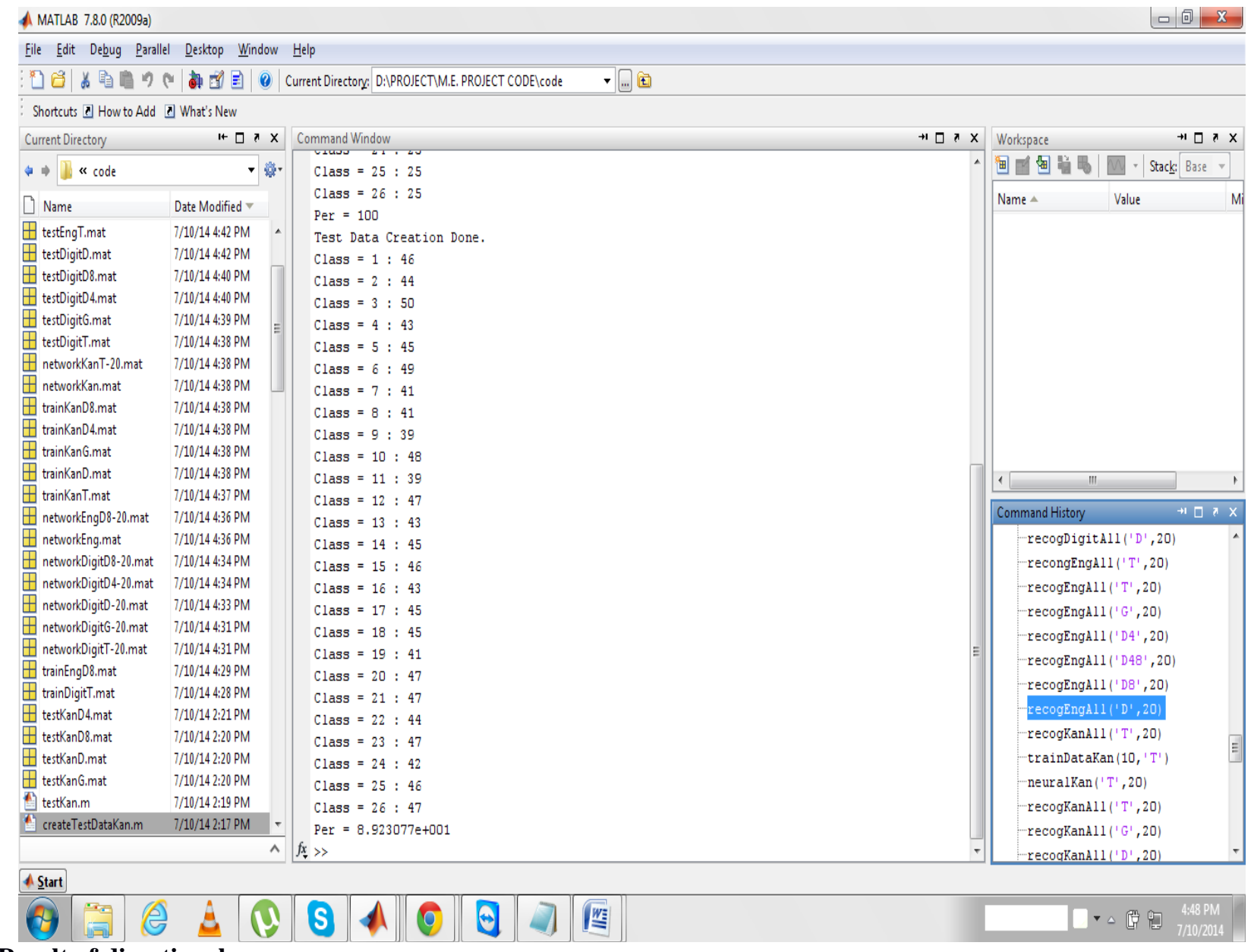

Result of directional

\begin{tabular}{|l|l|c|l|}
\hline Rate & Traditional & gradient & directional \\
\hline Recognition rate & $67 \%$ & $90 \%$ & $90 \%$ \\
\hline
\end{tabular}

\section{CONCLUSIONS}

This paper proposes about of directional feature extraction using the back propagation neural network. Before this work carried by using twelve direction on English character depending upon the four, eight and twelve directions without using neural network This paper is propose for Kannada digits, English and digits characters by using Traditional, directions, Gradient and comparing the results using neural network .

\section{REFERENCES}

1. Xin Wang, Ting-lei Huang, Xiao-yu Liu," Handwritten Character Recognition Based on BP Neural Network”, 978-0-7695-3899, 2009.

2. Vanita mane,lena gupta, "Handwritten character recognition using elastic matching and pca", international conference on advance in computing,communication and control ,ICAC3 '09, January 29-24, 2009.

3. Anita Pal, Dayashankar Singh," Handwritten English character recognition using using compass operator as feature extraction", International Journal of Advance Science \& Technology, ISSN 22295216 vol. 2. no. 4, 2011.

4. Bindu S Moni,G Raju "Modified quadratic classifier and directional features for handwritten Malayalam character recognition",Computational Science-New Dimensions and Perspectives, NCCSE,2011

5. Hailong Liu and Xiaoqing Ding," Handwritten Character Recognition Using Gradient Feature and Quadratic Classifier with Multiple Discrimination Schemes, 1520-5263/05, ICDAR'05

6. Anita Pal, Dayashankar Singh," Handwritten English character recognition using neural network", International Journal of Computer Science \& Communication IJCSC July-December 2010. Vol.1. No 2 ,2010

7. Jianchang Mao and Anil K. Jain, Fello," Introduction to artificial neural networks", 1996

8. Robert Hecht-Nielsen.'Theory of back propagation neural network"

9. Alexander J. Faaborg, , Using neural networks to create an adaptive character recognition system," May 2002

10. Simon Haykin."Feed forward neural networks an introduction" 
UGC Approved Journal

IJIREEICE

11. Jianchang Mao and Ani1 K. Jain, FelloHv, "Artificial Neural Networks for Feature Extraction and Multivariate Data Projection", IEEE Transactions on neural networks.1045-9227/95, vol 6. no. 2. march 1995

12. [Cheng-Lin Liu, "Normalization-cooperated gradient feature extraction for handwritten character recognition", IEEE Transactions on Pattern Analysis and Machine Intelligence, 0162-8828/07vol.29.no.8,August 2007.

13. Hiromichi fujisawa and cheng-lin liu," Directional pattern matching for character recognition revisited", international conference on document analysis and recognition.0-7695-1960, 2003 IEEE.

14. Weipeng zhang, yuan yan tang , and yun xue," Handwritten character recognition using combined gradient and wavelet feature,"1-424406056,2006 IEEE.

15. Robert E. using” Introduction to artificial neural networks," 0-7803-3026-919, 1995 IEEE.

\section{BIOGRAPHIES}

Ms. Shraddha Kunkunkar, Lecture in department of Electronics and Telecommunication, B.E (Electronics and Telecommunication) Mumbai University, Pursuing Masters of Engineering in Electronics and Telecommunication, Research interest in optical fiber communication and Image processing.

Mr. P. P. Narwade, Lecture in department of Electronics and Telecommunication, M.E (Biomedical Engineering), Mumbai University, Research interest in embedded system. 